Bio-grafía. Escritos sobre la Biología y su Enseñanza. ISSN 2027-1034

Número Extraordinario. p.p. 106-116

Memorias del Primer encuentro ambiental Universidad, ambiente y sustentabilidad: experiencias y prácticas.

\title{
LA FITORREMEDIACIÓN COMO ESTRATEGIA PARA REDUCIR IMPACTOS DEL MERCURIO EN AGUA: UN MICROPROYECTO DE EDUCACIÓN EN QUÍMICA VERDE
}

\author{
Alejandra Perdomo ${ }^{1}$ \\ María Velasco ${ }^{1}$ \\ María Cortés ${ }^{1}$ \\ Jhon Gallo ${ }^{1}$ \\ Ricardo Franco ${ }^{2}$
}

\section{Resumen}

En este trabajo se presenta el diseño y la aplicación de un sistema acuífero artificial a micro escala, contaminado con metales pesados como el mercurio, por medio del cual se pretende evaluar qué tan viable es la implementación de la fitoremediación como una tecnología limpia sustentable para disminuir el impacto ambiental negativo generado por la carga contaminante de metales pesados como el mercurio en ecosistemas acuáticos. En tal sentido, este trabajo se orienta desde los principios de la química verde, dejando a disposición de la comunidad académica de educadores en química, una estrategia para la enseñanza de esta disciplina, implementando dichos principios.

\section{Palabras Clave}

Fitoremediación, metales pesados, ecosistemas acuáticos, principios de química verde, educación química.

\section{Abstract}

In this work, we present design and application of a micro scale aquifer system contaminated with heavy metals such as mercury is carried out, by means of which it is tried to evaluate how feasible is the implementation of phytoremediation as a sustainable clean technology to reduce the negative environmental impact generated by the pollutant load of heavy metals

${ }^{1}$ Universidad Pedagógica Nacional. - Profesores en formación inicial - Departamento de Licenciatura en Química de la Universidad Pedagógica Nacional. - Microproyecto de investigación exploratoria desarrollado en el marco del curso electivo: "Química verde y energías alternativas para profesores de ciencias".

${ }^{2}$ Universidad Pedagógica Nacional - Profesor del Departamento de Licenciatura en Química. Semillero de Investigación DIDAGOKHEMIA. Grupo IREC. rfranco@ pedagogica.edu.co 
Bio-grafía. Escritos sobre la Biología y su Enseñanza. ISSN 2027-1034

Número Extraordinario. p.p. 106-116

Memorias del Primer encuentro ambiental Universidad, ambiente y sustentabilidad: experiencias y prácticas.

such as mercury in aquatic ecosystems. In this sense, this work is oriented from the principles of green chemistry, leaving to the academic community of educators in chemistry, a strategy for the teaching of this discipline, implementing these principles.

\section{Key Words}

Phytoremediation, heavy metals, aquatic ecosystems, green chemistry principles, chemical education

\section{INTRODUCCIÓN Y OBJETIVOS}

En este trabajo se presenta el desarrollo del microproyecto titulado "la fitoremediación como estrategia para reducir impactos del mercurio en agua", realizado en el espacio electivo Química verde y energías alternativas para profesores de ciencias del departamento de Química de la Universidad Pedagógica Nacional ofertado en el segundo semestre del año 2015; el proyecto surge a causa de la emergente problemática ambiental que abruma los recursos hídricos y los ecosistemas de agua dulce, surgiendo así la necesidad de proponer alternativas que conduzcan a la sustentabilidad ambiental y a disminuir el impacto ambiental.

La fitoremediación es una alternativa conveniente para reducir o degradar compuestos contaminantes en el suelo, el agua o el aire que provienen de las actividades cotidianas del ser humano, ya que se basa principalmente en la interacción de las plantas, el suelo y algunos microorganismos; además de esto, otras de las ventajas de usar esta técnica, es que tiene un costo económico bajo y los procesos que se realizan desde el uso de plantas son más ecológicos en los ecosistemas. Dentro de este término se encuentran una serie de fitotecnologías que contienen mecanismos fisiológicos para la recuperación de ecosistemas contaminados, por lo tanto, la realización de este microproyecto se orientó a partir de la siguiente pregunta: ¿Cuál es la importancia de realizar un micro-proyecto de química verde, vinculando la fitoremediación con miras a la mitigación del impacto de metales pesados como el mercurio en fuentes hídricas? Así, se pretende analizar la importancia de la fitoremediación como una técnica de química verde para disminuir el impacto ambiental que genera el mercurio $(\mathrm{Hg})$ en fuentes hídricas, a partir del estudio de la planta 
Bio-grafía. Escritos sobre la Biología y su Enseñanza. ISSN 2027-1034

Número Extraordinario. p.p. 106-116

Memorias del Primer encuentro ambiental Universidad, ambiente y sustentabilidad: experiencias y prácticas.

"Eichhornia crassipes (Jacinto de agua)" con el fin de determinar la propiedad de absorción que tiene la planta frente a dicho metal en un ecosistema acuático y, por último, establecer la relación entre la técnica fotoquímica de la fitoremediación y la química verde.

\section{MARCO DE TEORICO}

\section{Generalidades}

La contaminación por metales pesados se ha constituido en una de las formas más peligrosas para los ecosistemas acuáticos, dado que son elementos poco o nada biodegradables, tienden a acumularse en los tejidos de animales y vegetales acuáticos, y permanecen en ellos por largos períodos, desencadenando procesos de biomagnificación y acciones toxico dinámica, las cuales generan alteraciones metabólicas, mutaciones y transformaciones anatómicas en las especies animales, incluido el hombre. Las altas concentraciones de los metales en el medio acuático y sus componentes (agua, sedimentos, flora y fauna, entre otros) son provocadas principalmente por actividades antropogénicas directas o indirectas, sobre ese medio (Marrugo, 2011).

Los metales pesados tienen tres vías principales de entrada a los sistemas acuáticos:

- La vía atmosférica, se produce debido a la sedimentación de partículas emitidas a la atmósfera por procesos naturales o antropogénicos, principalmente combustión de combustibles fósiles y procesos de fundición de metales.

- La vía terrestre, es producto de filtraciones de vertidos, de la escorrentía superficial de terrenos contaminados (minas, utilización de lodos como abono, lixiviación de residuos sólidos, precipitación atmosférica, etc.) y otras causas naturales.

- La vía directa, es a consecuencia de los vertidos directos de aguas residuales industriales y urbanas a los cauces fluviales.

La fitoremediación y plantas hiperacumuladoras. 
Bio-grafía. Escritos sobre la Biología y su Enseñanza. ISSN 2027-1034

Número Extraordinario. p.p. 106-116

Memorias del Primer encuentro ambiental Universidad, ambiente y sustentabilidad: experiencias y prácticas.

El término fitoremediación hace referencia a una serie de tecnologías que se basan en el uso de plantas para limpiar o restaurar ambientes contaminados, como aguas, suelos, e incluso aire. Es un término relativamente nuevo, acuñado en 1991 (Agudelo et al. 2005). Se compone de dos palabras, Fito, que en griego significa planta o vegetal, y remediar (del latín remediare), que significa poner remedio al daño, o corregir o enmendar algo. Fitoremediación significa remediar un daño por medio de plantas o vegetales.

\section{Fitoremediación acuática.}

Tradicionalmente, las plantas vasculares acuáticas han sido consideradas como una plaga en sistemas enriquecidos con nutrientes (Olguín, 2013). Su rápida proliferación puede dificultar la navegación y amenazar el balance de la biota en los ecosistemas acuáticos. Sin embargo, en la actualidad se considera que estas plantas también pueden ser manejadas adecuadamente y volverse útiles, debido a su capacidad para remover y acumular diversos tipos de contaminantes. Además, su biomasa puede ser aprovechada como fuente de energía, forraje y fibra (Lascano 2014).

\section{Criterios de selección de plantas para la fitoremediación.}

La eficiencia de remoción de contaminantes durante el proceso de fitoremediación dependerá principalmente de la especie de planta utilizada, el estado de crecimiento de las plantas, su estacionalidad y el tipo de metal a remover. Por lo mismo, para lograr buenos resultados, las plantas a utilizar deben tener las siguientes características:

- Ser tolerantes a altas concentraciones de metales.

- Ser acumuladoras de metales.

- Tener una rápida tasa de crecimiento y alta productividad.

- Ser especies locales, representativas de la comunidad natural.

- Ser fácilmente cosechables.

\section{Eichhornia crassipes}


Bio-grafía. Escritos sobre la Biología y su Enseñanza. ISSN 2027-1034

Número Extraordinario. p.p. 106-116

Memorias del Primer encuentro ambiental Universidad, ambiente y sustentabilidad: experiencias y prácticas.

Atehortua y Gartner (2013) indican que la Eichhornia crassipes es una planta macrófita de la familia de las monocotiledóneas. Es conocida como Buchón de Agua. Está constituida por un tejido de polisacáridos estructurales como celulosa y hemicelulosa, además de lignina, un polímero aromático, heterogéneo e insoluble en ácidos. De acuerdo con Benítez et al. (2011) es una especie flotante de raíces sumergidas. Carece de tallo aparente, provisto de un rizoma, muy particular, emergente, del que se abre un rosetón de hojas que tienen una superficie esponjosa notablemente inflada en forma de globo y forma una vejiga llena de aire mediante la que el vegetal puede mantenerse sobre la superficie acuática.

\section{Química verde}

En Estados Unidos surge la Agencia de Protección Ambiental (EPA por su sigla en inglés), con el objetivo de cuidar la salud humana y el medio ambiente (a principios de la década de los noventa, los químicos Paul Anastas y John Warner que trabajaban para la EPA, proponen el concepto de Química Verde para referirse a aquellas tecnologías químicas que apuntan a prevenir la contaminación (Pájaro y Tadeo 2011)

La química verde en general busca procesos para obtener los mismos productos que la química tradicional de manera menos contaminante y también producir sustancias no contaminantes que sustituyan a otros productos perjudiciales pero necesarios para la sociedad, Su metodología se basa en 12 principios propuestos por el propio Anastas y Warner.

\section{METODOLOGÍA}

Para el desarrollo experimental, se optó por un análisis cuantitativo, puesto que se buscó cuantificar la concentración del metal traza $(\mathrm{Hg})$ acumulado por la especie vegetal Eichhornia crassipes (Jacinto de agua), en el cuerpo de agua simulado.

\section{Diseño experimental}


Bio-grafía. Escritos sobre la Biología y su Enseñanza. ISSN 2027-1034

Número Extraordinario. p.p. 106-116

Memorias del Primer encuentro ambiental Universidad, ambiente y sustentabilidad: experiencias y prácticas.

1. La planta fue lavada con agua destilada para eliminar resto de sedimentos, en el caso de que la planta presentase tejido dañado o deteriorado, éste fue descartado para el ensayo.

2. La planta seleccionada fue colocada en recipientes plásticos con $100 \mathrm{ml}$ de una solución de $\mathrm{HgO}$ (II) a una concentración de 5 ppm.

3. El experimento consistió en un diseño completamente aleatorio. Se tomaron muestras de $5 \mathrm{ml}$ de la solución a las $0,1,1: 30,2,3$, horas de incubación para cada tratamiento y control.

4. Al final del experimento la planta fue retirada de los recipientes con la solución, lavada con agua y colocada en una bolsa de residuos peligrosos, para su debido tratamiento

5. La concentración de metal en la solución fue determinada por espectrofotometría de absorción atómica, mediante la técnica de generador de hidruros. El método de borohidruro de sodio implica la reacción del analito en una solución ácida $(\mathrm{HCl})$, con el $\mathrm{NaBH}_{4}$ para formar hidruro gaseoso del elemento en estudio que en este caso es el Mercurio. De igual forma, se realizó un análisis de regresión para comparar las curvas de concentración de $\mathrm{Hg}$ en solución en función del tiempo.

\section{RESULTADOS}

\section{Características organolépticas:}

El Jacinto de agua es una maleza acuática perenne que flota libremente en la superficie del agua. Esta planta es nativa de Brasil y fue introducida a EU en 1884 como planta ornamental para jardines acuáticos. La reproducción del Jacinto de agua ocurre principalmente de forma vegetativa por medio de la producción de estolones. La producción de semillas también ocurre, aunque con un bajo porcentaje de germinación. Bajo temperaturas optimas de crecimiento, la biomasa del Jacinto de agua puede duplicarse en un mes por medio de reproducción vegetativa. Esta alta capacidad reproductiva provoca la formación de colonias densas flotando en el agua.

Para la práctica se distribuyó la planta de la especie Jacinto de agua en el montaje para la adsorción de $\mathrm{Hg}$ (II) en concentración de 5 ppm, como resultado se evidencia que al cabo de las 72 horas la planta presenta una 
Bio-grafía. Escritos sobre la Biología y su Enseñanza. ISSN 2027-1034

Número Extraordinario. p.p. 106-116

Memorias del Primer encuentro ambiental Universidad, ambiente y sustentabilidad: experiencias y prácticas.

coloración café en las hojas y en su rizoma (ver imagen 2), lo cual indica la absorción de mercurio y la distribución a sus órganos.

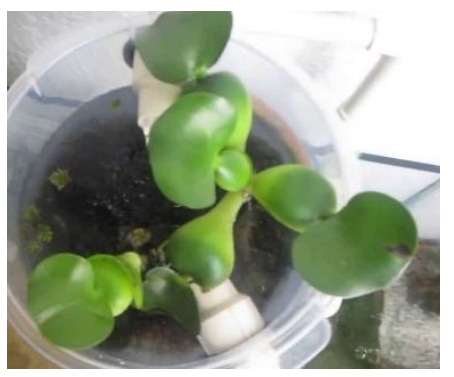

Imagen 1. Eichhornia crassipes en tiempo 0 Eichhornia crassipes después de

a la solución de $\mathrm{HgO}$.

\section{Remoción de $\mathrm{Hg}$ en solución.}

La concentración remanente de mercurio en la solución, el porcentaje de remoción y de retención de la especie, se encuentran en la tabla 2.

\begin{tabular}{|c|c|c|c|c|}
\hline $\begin{array}{c}\text { Tiempo } \\
(\mathrm{h})\end{array}$ & Absorbancia & {$\left[\mathrm{Hg}^{2+}\right] \mathrm{ppb} /$ muestra } & $\begin{array}{c}\% \\
\text { Remoció } \\
\mathrm{n}\end{array}$ & $\begin{array}{c}\% \\
\text { Retención }\end{array}$ \\
\hline 0 & 0,034 & 36,12 & 0,722 & 99,277 \\
\hline 1 & 0,054 & 61,12 & 1,222 & 98,776 \\
\hline $1: 30$ & 0,167 & 202,3 & 4,046 & 95,954 \\
\hline 3 & 0,094 & 111,12 & 2,222 & 97,777 \\
\hline
\end{tabular}

Tabla 2. Porcentaje de Retención y Remoción de mercurio por la especie vegetal.

En la tabla 3 se observa que la concentración de mercurio absorbido por la especie vegetal conlleva un proceso de bioacumulación, ya que se presenta un aumento de la concentración de $\mathrm{Hg}^{+2}$ en el organismo biológico, en el lapso entre 0 y 1:30 (una hora y treinta minutos). Cuando se llega al tiempo final 3 horas se observa que la concentración de $\mathrm{Hg}^{+2}$ con respecto a el tiempo 1:30 hora y treinta minutos, descendió, en este punto puede deducirse que las plantas se sobresaturan en este tiempo. El porcentaje de remoción nos indica la cantidad de $\mathrm{Hg}$ presente en la solución de 5 ppm al cabo de; tiempo 0,1 hora, 1 hora treinta minutos y 3 
Número Extraordinario. p.p. 106-116

Memorias del Primer encuentro ambiental Universidad, ambiente y sustentabilidad: experiencias y prácticas.

horas. En el tiempo de 3 horas la especie vegetal deja de remover el metal de la solución de 5 ppm y empieza de devolver parte del contaminante de nuevo a la solución, y deja de retener el metal pasando de una retención de un $95,9 \%$ en tiempo una hora treinta minutos a un $97,77 \%$. Al determinar el \% de remoción y el \% retención en el muestreo con intervalo se observa la eficacia de la especie que contrastada con la teoría es coherente a los estudios presentados por Benítez et al. (2011) en donde manifiestan que el aumento de la concentración del metal en el tejido de la planta correspondió a una disminución en el medio, esta exposición tan prolongada generalmente aumenta la condición de estrés de la planta, que podría conducir a liberar parte de la carga de metal al medio, manteniendo con ello la regulación homeostática mediante mecanismos que permiten una menor afinidad de los sitios de adhesión por los metales o empleando mecanismos que detectan de manera rápida la presencia del metal, facilitando su excreción activa, o menor permeabilidad de la membrana celular. La acumulación de metales pesados por parte de especies vegetales consiste primero en una absorción rápida, seguido del transporte lento, controlado principalmente por la difusión al interior del celular (Bioacumulación), esto explica por qué inicialmente la planta empieza a retener y remover un mayor porcentaje $\mathrm{Hg}$ de la solución de 5 ppm.

\section{CONCLUSIONES}

La iniciativa de implementar la fitoremediación como alternativa para disminuir el impacto de metales pesados tales como el mercurio (II) es buena en la medida en la cual se exponga la planta Jacinto de agua en los tiempos determinados para remover el mercurio, de lo contrario la planta después de un intervalo determinado de tiempo se sobresatura del metal y expulsa de nuevo el mercurio al medio.

La realización de microproyectos que aborden temas de química verde es fundamental en la formación inicial de profesores de ciencias ya que de esta manera se logra tener un conocimiento más específico frente a las cuestiones ambientales que nos rodean a diario y por esto se tendrá la capacidad de propagar esta información en nuestras aulas de clase para cumplir con una labor ambiental que nos compete a todos. 
Bio-grafía. Escritos sobre la Biología y su Enseñanza. ISSN 2027-1034

Número Extraordinario. p.p. 106-116

Memorias del Primer encuentro ambiental Universidad, ambiente y sustentabilidad: experiencias y prácticas.

En cuanto a las oportunidades de mejoramiento, por medio de este microproyecto se logró evidenciar la importancia que tiene el uso de métodos de análisis químico, ya que de esta manera es posible viabilizar a microescala una alternativa que luego se puede realizar a microescala para disminuir algún impacto ambiental en el caso de este proyecto la mitigación del mercurio (II) en fuentes hídricas. De igual modo, existe un amplio riesgo ambiental al hacer la preparación de una solución de mercurio (II) para realizar el microproyecto, ya que si no se tienen las medidas de manipulación necesarias se podría contaminar alguna fuente hídrica o el ambiente. En este caso no se cumple el principio de prevención el cual afirma que es mejor prevenir la generación de un residuo, que tratarlo o eliminarlo después de haberlo generado.

Es importante buscar alternativas eficientes para tratar la biomasa generada en el proceso de remediación, porque se estaría resolviendo un problema de contaminación, pero provocando otro, ya que en términos reales lo único que está sucediendo es la transferencia de metales de una fase acuosa (agua contaminada) a una fase sólida (biomasa contaminada).

\section{REFERENCIAS BIBLIOGRÁFICAS}

Agudelo, L. et al. (2005). Fitoremediación: la alternativa para absorber metales pesados de los biosólidos. Revista lasallista de investigación vol. $2 . \quad$ Disponible en: http://www.lasallista.edu.co/fxcul/media/pdf/Revista/vol2n1/fitorremediacio $\underline{\text { n.pdf }}$

Atehortua, E. y Gartner, C. (2013). Estudios preliminares de la biomasa seca de Eichhornia crassipes como adsorbente de plomo y cromo en aguas. Revista Colombiana de Materiales, (4), 8192.Disponible en: https://webcache.googleusercontent.com/search?q=cache: wUdP BP0P wJ:https://aprendeenlinea.udea.edu.co/revistas/index.php/materiales/articl e/download/15084/13161+\&cd=1\&hl=es\&ct=clnk\&gl=co

Benítez, R., Calero, V., Peña, E. y Martin, J. (2011). Evaluación de la cinética de la acumulación de cromo en el buchón de agua (Eichhornia crassipes). Biotecnología en el sector agropecuario y agroindustrial, 9(2), pp.

66-73

Recuperado

de 
Bio-grafía. Escritos sobre la Biología y su Enseñanza. ISSN 2027-1034

Número Extraordinario. p.p. 106-116

Memorias del Primer encuentro ambiental Universidad, ambiente y sustentabilidad: experiencias y prácticas.

http://www.scielo.org.co/scielo.php?script=sci arttext\&pid=S1692$\underline{35612011000200008}$

Delgadillo, A. et al. (2011). Fitoremediación: una alternativa para eliminar la contaminación. Universidad Autónoma del Estado de Hidalgo, Instituto de Ciencias Básicas e Ingeniería, Centro de Investigaciones Químicas. Consulta:

01/09/2005.

Disponible

en:

http://www.uaeh.edu.mx/investigacion/icap/LI IntGenAmb/Otilio Sando/1. pdfhttp://www.uaeh.edu.mx/investigacion/icap/LI_IntGenAmb/Otilio_Sando 11.pdf

Galán, E y Romero, A. (2008). Contaminación por metales pesados. Departamento de Cristalografía, Mineralogía y Química Agrícola. Facultad de Química. Apartado 553. Universidad de Sevilla. Sevilla 41071. Consulta 27/08/2015. Disponible en: http://www.ehu.eus/sem/macla pdf/macla10/Macla10 48.pdf

Lascano, A. (2014). Fitoremediación de aguas residuales. Revisado el 01 de octubre de 2015.2 Disponible en: https://prezi.com/51qutnpljo3a/fitorremediacion-de-aguas-residuales/

Marrhttp://www.ehu.eus/sem/macla_pdf/macla10/Macla10_48.pdfhttp://ww w.ehu.eus/sem/macla_pdf/macla10/Macla10_48.pdfugo, J. (2011). Evaluación de la contaminación por metales pesados en la ciénaga la soledad y bahía de cispatá, cuenca del bajo sinú, departamento de córdoba. Grupo de Investigación en Aguas, Química Aplicada y Ambiental - GAQAA. Disponible en: http://web.www3.unicordoba.edu.co/sites/default/files/Informe\%20Final $\mathrm{F}$ CB\%2010-08\%20Jos\%C3\%A9\%20Luis\%20Marrugo\%20Negrete.pdf

Núñez, R. et al. (2004). Fitoremediación Fundamentos y aplicaciones. Consulta: 27/08/2015. Disponible en: http://datateca.unad.edu.co/contenidos/301332/CORE 2013/Actividad 7.p dfhttp://datateca.unad.edu.co/contenidos/301332/CORE_2013/Actividad_7 .pdf

Olguín, E. (2013). Fitoremediación Acuática. Instituto de ecología. México. Consulta 27/08/2015.Disponible en : http://www.iingen.unam.mx/es$\underline{\mathrm{mx} / \text { BancoDelnformacion/MemoriasdeEventos/SolucionesContaminacionS }}$ 
Bio-grafía. Escritos sobre la Biología y su Enseñanza. ISSN 2027-1034

Número Extraordinario. p.p. 106-116

Memorias del Primer encuentro ambiental Universidad, ambiente y sustentabilidad: experiencias y prácticas.

uelo/EugeniaOlguinPalacios.pdfhttp://www.iingen.unam.mx/es-

$\mathrm{mx} /$ BancoDelnformacion/MemoriasdeEventos/SolucionesContaminacions uelo/EugeniaOlguinPalacios.pdf

Pájaro, A. y Tadeo, J. (2011). Química verde: un nuevo reto, Green chemistry: a new challenge. consulta:06/09/2015.

Disponible

en:

http://www.scielo.org.co/scielo.php?script=sci arttext\&pid=S0124-

$\underline{81702011000200009}$

Poma, V. y Valderrama, A. (2014). Estudio de los parámetros fisicoquímicos para la fitoremediación de cadmio (ii) y mercurio (ii) con la especie Ei chhornia Crassipes (Jacinto de agua). Rev. Soc. Quím. Perú .2014, vol.80, n.3, pp. 164-173. Consulta: 01/09/2015 Disponible en: http://www.scielo.org.pe/scielo.php?pid=S1810-

634X2014000300003\&script=sci abstract 